\title{
TAIGA-Observatory: First 5 years of operation of the HiSCORE Air-Cerenkov Array
}

\section{Andrea Porelli ${ }^{a, *}$ on behalf of the TAIGA Collaboration}

(a complete list of authors can be found at the end of the proceedings)

${ }^{a}$ DESY-Zeuthen, Platanenallee 6, 15738, Zeuthen, Germany

E-mail: andrea.porelli@desy.de

HiSCORE is a wide-aperture Air-Cherenkov array, and is a major component of the TAIGAObservatory (Tunka Advanced Instrument for cosmic ray physics and Gamma Astronomy), located in the Tunka valley, $50 \mathrm{~km}$ from Lake Baikal, Russia. A main science target of TAIGA is gamma ray astronomy above ten's of $\mathrm{TeV}$, in particular the search for sources of few $100 \mathrm{TeV}$ gamma rays (candidate "PeVatrons"), the possible sites of Galactic cosmic ray acceleration. The HiSCORE array will consist of 120 optical Cherenkov stations, deployed on an area of $1 \mathrm{~km}^{2}$. Its construction will be finished in 2021.

This report presents the performance of HiSCORE during its first 5 years of operation, in various configurations, from 28 to 89 stations. A key for high sensitivity to gamma point sources is precision timing of the whole array down to sub-nsec level, required to be stable for the observation period. We apply different methods to reach this goal. The pointing resolution of the array for extended airshowers is obtained as $0.1^{\circ}$ for highest energies, and is experimentally verified, based on independent approaches. We present results of a 5-year-search for gamma-like point sources with HiSCORE in stand-alone operation.

$37^{\text {th }}$ International Cosmic Ray Conference (ICRC 2021)

July 12th-23rd, 2021

Online - Berlin, Germany

\footnotetext{
*Presenter
} 


\section{Introduction}

TAIGA-HiSCORE [1] is a wide-aperture Air-Cherenkov array, and is a major component of the TAIGA-Observatory (Tunka Advanced Instrument for cosmic ray physics and Gamma Astronomy), located in the Tunka valley, $50 \mathrm{~km}$ from Lake Baikal, Russia [2]. A main science target of TAIGA is gamma ray astronomy above ten's of TeV, in particular the search for sources of few $100 \mathrm{TeV}$ gamma rays (candidate "PeVatrons"), the possible sites of Galactic cosmic ray acceleration. The final stage of the TAIGA prototype array (figure 1(a)) will be ready by end of 2021, and will count 120 TAIGAHiSCORE stations, deployed on an area of $1 \mathrm{~km}^{2}, 2(+1$ in construction) operating TAIGA-IACTs, and sparse surface/underground TAIGA-Muon detectors $\left(240 \mathrm{~m}^{2}\right)$.

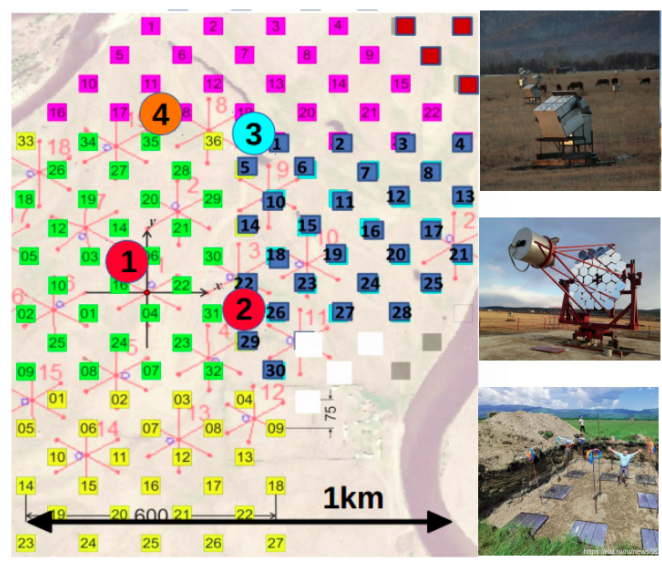

(a)

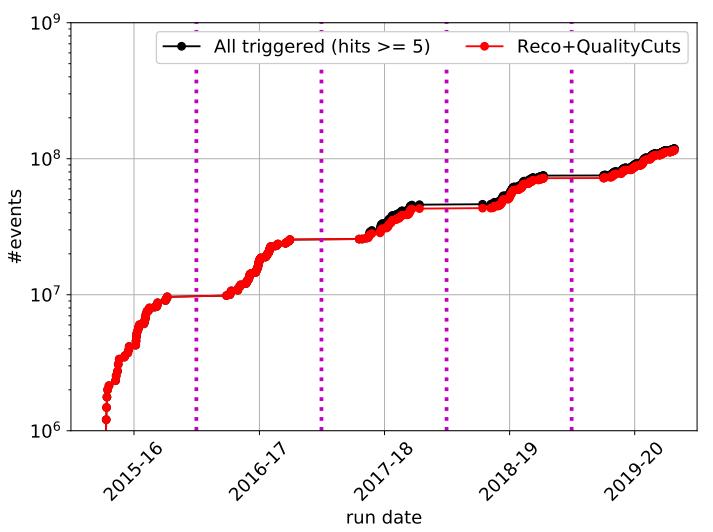

(b)

Figure 1: TAIGA Observatory. (a) TAIGA prototype array layout planned for fall 2021: 120 HiSCORE array stations, 2(+1 in construction) operating IACT telescopes. A sparse surface/underground TAIGA-Muon detectors $\left(240 \mathrm{~m}^{2}\right)$ will start operation soon. (b) TAIGA-HiSCORE integrated number of triggered events (\#hits $\geq 5$, black), and after quality cuts (red), over the first 5 years of operation.

HiSCORE started its stable operation in 2015/16 with 1 cluster (28 stations, extended to 32 in 2017/18). The second cluster (27 stn.) started operation in 2017/18, and the third cluster (30 stn.) in January 2020, for a total of 89 stations. A fourth cluster ( 30 stn.) is currrently under construction, and will start operating by the end of 2021. Figure 1(b) shows the integrated number of detected events $\left(\geq 10^{8}\right)$ during the first 5 years of operation.

\section{Air shower reconstruction}

The main role of HiSCORE in TAIGA is to provide accurate shower core and direction reconstruction, as well as shower energy. The standard EAS reconstruction procedure $[3,4]$ is done in two steps: a first approximation of the shower core is obtained from a weighted average of the registered signal amplitude, while a plane wave fit of the pulse arrival time provides a first estimation of the 
shower direction. If signal from more than 5 stations is available, a more precise reconstruction of the core is possible by fitting the amplitude distribution as function of the core distance (ADF), while for the direction, a fit of the shower front with a parabolic (PAR) model is performed. Figure 2(a) gives an example of a reconstructed HiSCORE events (1 cluster array), while figure 2(b) shows the core and arival direction acceptance for HiSCORE operating with 3 cluster (no quality cuts). Section 2.1 will present the array time calibration adopted to achieve the require $0.1^{\circ}$ angular resolution (for events with large multiplicity/energy), while section 2.2 will present the method used to experimentally verify the detector angular resolution.
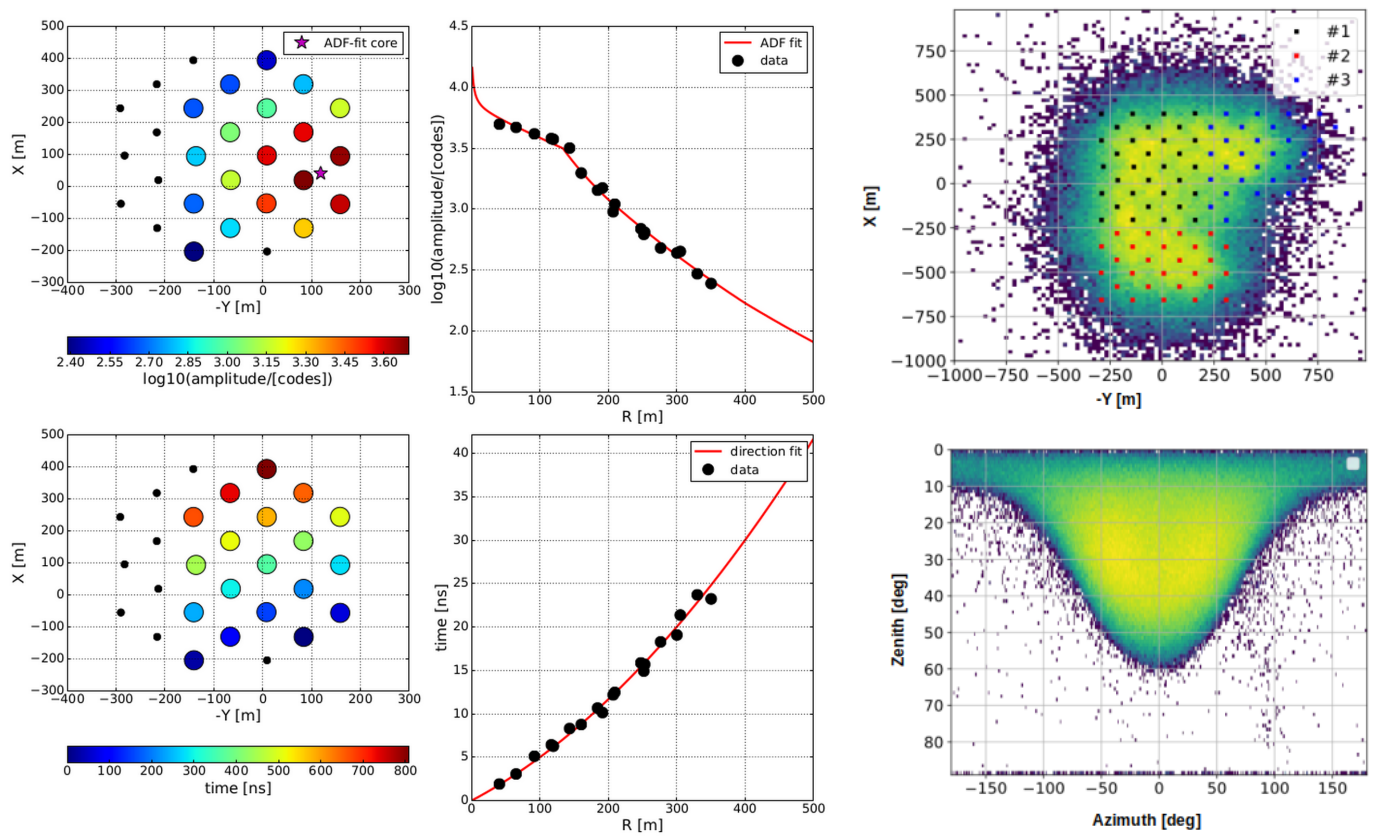

(a)

(b)

Figure 2: HiSCORE EAS reconstruction: (a) Example of a reconstructed event (1 cluster). Top-left: station pulse amplitudes distribution and reconstructed core position (purple star). Top-right: amplitude distribution as function of the distance from the shower axis. Bottom-left: station pulse arrival time distribution. Bottom-right: shower front arrival time as function of the distance from the shower axis. Red lines: ADF and PAR fit results. (b) Reconstructed cores (top) and arrival directions (bottom) distribution for a 3 cluster HiSCORE array (no quality cuts).

\subsection{Array time calibration}

To achieve the required angular resolution, a sub-nsec time synchronization between the stations is required [3], which implies a precise measurement of the station timing. In HiSCORE, PMTs and electronics in the station introduce some unknown time offsets, which affect the detector angular resolution and the absolute pointing precision. For this work, an accurate estimation of such time 
offsets is obtained with the Hybrid calibration method (HYB) [5, 6], which is an iterative procedure that combines information from EAS detected all over the array, and information from few stations calibrated with external LED, following an approach established for ARGO-YBJ [7].

Figure 3(a) shows the estimated station time offsets obtained for season 2015/16 using the HYB method. The color code indicates the different PMT types operating in the optical modules. Despite using only one LED calibration per observation season, the calibration results are very stable over time. To verify the efficiency of the calibration, we analyze the direction reconstruction fit residual distribution, presented in figure 3(b). The calibrated data (black dost) nicely agree with MC prediction for ideal case with no time offsets (black line). For comparison, the same distribution from uncalibrated data is shown (grey crosses). A $0.57 \mathrm{~ns}$ rms is obtained, proving the calibration efficiency and the sub-ns time synchronization between the stations.

As shown in detail in [6], the test with residual distributions only proves that the detector can achieve the required angular resolution (see section 2.2). To verify the array absolute pointing, and thus the full efficiency of the calibration method, an external source is needed. In HiSCORE, such a test is performed using satellite LIDARs $[6,8,9]$, proving a residual mispointing of $\sim 0.1^{\circ}$.

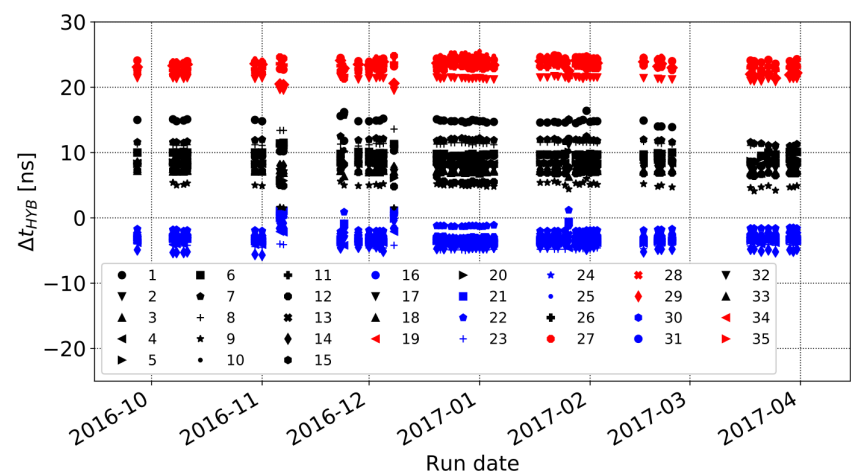

(a)

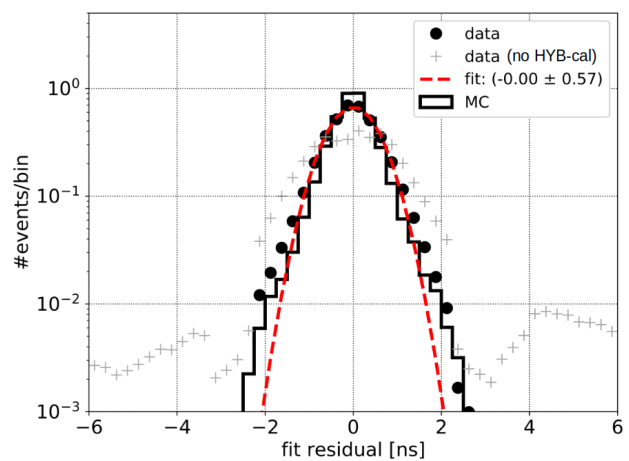

(b)

Figure 3: HiSCORE time calibration: Hybrid method. (a) Station time offsets as function of run date (x-axis) and station ID (marker). The color code indicates the different PMT types operating in the optical modules. (b) EAS direction reconstruction fit residual before (grey crosses) and after applying the HYB calibration (black). A $0.54 \mathrm{~ns}$ rms is obtained, proving the calibration efficiency and the sub-ns time synchronization between the stations.

\subsection{Angular resolution test: Chessboard method}

Beside using MC simulation, the HiSCORE angular resolution is experimentally determined using the chessboard method $[6,12]$. The array is split into two interspersed sub-arrays (like a chessboard, figure 4(a)), and each triggered shower is reconstructed using the two independent sub-arrays. The space angle between the two reconstructed directions, with good approximation, is twice the full-array angular error, $\psi$. Figure 4 (a) shows the detector angular error (at $72 \%$ containment) as function of the total number of triggered stations, for three different TAIGA-HiSCORE layouts: 1, 2, and 3 cluster. 


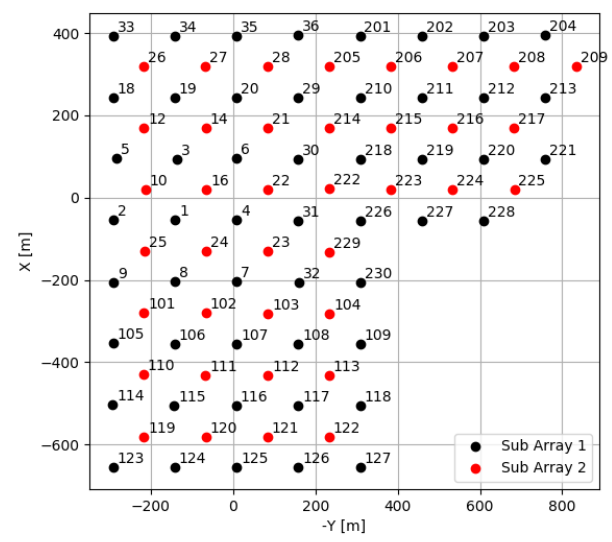

(a)

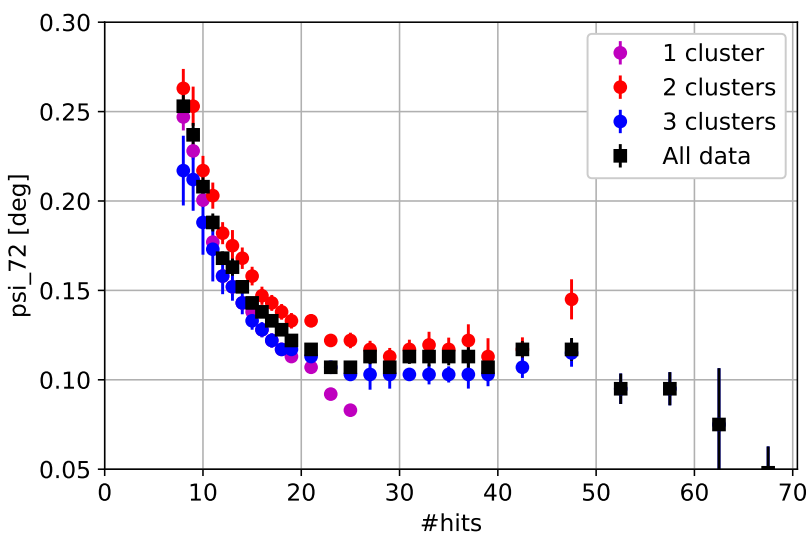

(b)

Figure 4: HiSCORE angular resolution: Chessboard method. (a) Chessboard-like splitting of the HiSCORE 3 cluster array. (b) Estimated angular resolution as function of total number of triggered stations: $\sim 0.2^{\circ}$ at multiplicity $\geq 10 ; \sim 0.1^{\circ}$ at multiplicity $\geq 20$.

In black, the result obtained using the full 5 years data sample. An error of $\sim 0.2^{\circ}$ is achieved at event multiplicity $\geq 10$, and $\sim 0.1^{\circ}$ at event multiplicity $\geq 20$.

\section{Point source analysis}

We developed a full-sky point source analysis [6] based on the Direct Integration method [11]. For this analysis, we select events which survive reconstruction quality cuts, and with number of hits $\geq 10$ (angular resolution $\leq 0.2^{\circ}$ ). Additionally, events from known space-born LIDARs signals (ISS/CATS, CALIPSO) are excluded [8, 9]. For each event filling the signal map, we fill the background map by generating 100 fake events with random time. Each bin in the maps is scaled by a factor $1 / \cos (\mathrm{d})$, and both maps are then smoothed using a uniform kernel of radius $0.3^{\circ}\left(\sim 1.58 \times 0.2^{\circ}\right)$. The significance for each bin is calculated using Li\&MA formula 14, with a background $\alpha$ factor of 0.01 [13].

Figure 5 presents the results obtained analysing the data collected by TAIGA-HiSCORE during its first 5 years of operation. Figure 5(a) shows a detail of the significance map, zoomed around the Crab direction (main test source). As expected, no source signal above $5 \sigma$ is observed, due to the large background. Besides the definition of efficient $\mathrm{g} / \mathrm{h}$ rejection cuts, an energy binned analysis, where smaller search radius can be used at higher energy, could improve the results. The full-sky significance distribution (figure 5(b), black) is well described by a Gaussian with $\mu=0$ and $\sigma=1$, and represents a good indication of correct background estimation. The detection potential of the analysis is demonstrated by the detection of few, previously unkown satellite LIDAR signals, as shown in figure 5(b) (grey dotted line, run 16/10/2015). 


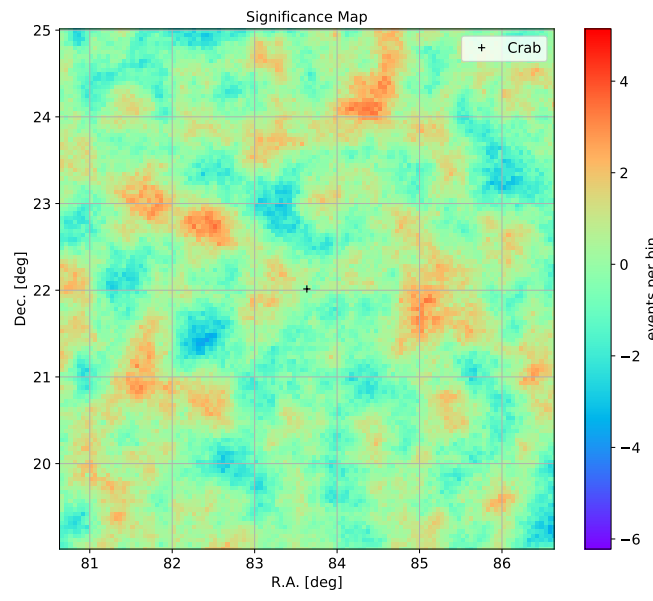

(a)

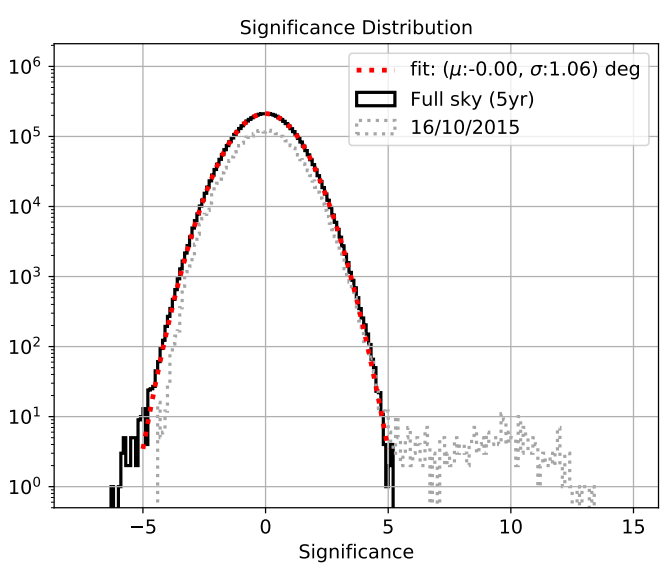

(b)

Figure 5: HiSCORE stand alone point source analysis. (a) Detail of full-sky significance map, zoomed around Crab. (b) Significance distribution: full-sky (black), and for a run containing undetected satellite LIDAR events (grey, run 16/10/2015). The significance distribution is well described by a Gaussian with $\mu=0$ and $\sigma=1$, giving a good indication for the correct background estimation.

\section{Conclusion}

The HiSCORE array is a major component of the TAIGA Observatory, in stable operation since fall 2015, providing precise EAS core and arrival direction reconstruction, with a wide angle sky exposure. We presented here results from standard calibration and reconstruction performed on the data collected in the first 5 years.

The Hybrid time calibration method allows efficient correction of the unknown station time offset, achieving a sub-nsec time synchronization between the stations, and thus obtaining the required angular resolution and absolute pointing. We used the Chessboard method to check experimentally the detector angular resolution, obtaining an error of $\sim 0.2^{\circ}$ for multiplicity $\geq 10$, and $\sim 0.1^{\circ}$ for multiplicity $\geq 20$. External satellite LIDAR signals are instead used to check the array absolute pointing $\left(\sim 0.1^{\circ}\right)$.

Finally, we performed a preliminary full-sky point source analysis, integrated over the full 5 years data set. We obtain a full-sky significance distribution which is well described by a Gaussian centred at 0 and width 1 , indicating a correct estimation of the background. While no gamma sources are detected above $5 \sigma$ due to the large hadrons background, the analysis revealed the presence of few undetected satellite LIDAR signals.

\section{Acknowledgments}

This work was performed at the UNU " Astrophysical Complex of MSU-ISU» (agreement 13.UNN.21.0007). The work is supported by Russian Foundation for Basic Research (grants 19-52- 
44002, 19-32-60003) the Russian Science Foundation (grant 19-72-20067 (Section ????), the Russian

Federation Ministry of Science and High Education (projects FZZE-2020-0017, FZZE-2020-0024).

\section{References}

[1] Tluczykont, M., Hampf, D., Horns, D., et al. (2011). Adv. Sp. Res., 48(12):1935-1941, arXiv:1108.5880.

[2] N. Budnev et al., PoS(ICRC2021)731

[3] D. Hampf et al. (2011). 25th Texas Symp. Relativ. Astrophys., arXiv:1104.2336.

[4] V. Prosin et al. (2009), Nucl. Phys. B - Proc. Suppl., 190(C):247-252.

[5] A. Porelli et al., PoS(ICRC2017)752

[6] A. Porelli, PhD thesis, Humboldt University (2020), http://edoc.hu-berlin.de/18452/22369

[7] He, H. H., Bernardini, P., Calabrese Melcarne, A. K., et al. (2007). Astropart. Phys., 27(6):528-532, arXiv:0701291.

[8] A. Porelli et al., PoS(ICRC2017)754

[9] A. Porelli et al., PoS(ICRC2017)752

[10] N. Budnev et al., Nuclear Inst. and Methods in Physics Research, A 958 (2020) 162113

[11] Alexandreas et al. (1993). Nucl. Instruments Methods Phys. Res. Sect. A Accel. Spectrometers, Detect. Assoc. Equip., 328(3):570-577.

[12] Karle, A. (1991).In AIP Conf. Proc., volume 220, pages 127-131. AIP.

[13] Li, T. P. and Ma, Y. Q. (1983). Astrophysical Journal, 272:317-324. 


\section{Full Authors List: TAIGA Collaboration}

I. I. Astapov ${ }^{2}$, A. K. Awad ${ }^{10}$, P. A. Bezyazeekov ${ }^{3}$, M. Blank ${ }^{10}$, E. A. Bonvech ${ }^{1}$, A. N. Borodin ${ }^{4}$, A. V. Bulan ${ }^{1}$, M. Brueckner ${ }^{5}$, N. M. Budnev $^{3}$, A. Chiavassa ${ }^{6}$, D. V. Chernov ${ }^{1}$, A. N. Dyachok ${ }^{3}$, A. R. Gafarov ${ }^{3}$, A. Yu. Garmash ${ }^{7,8}$, V. M. Grebenyuk ${ }^{4,9}$, O. A. Gress ${ }^{3}$, E.

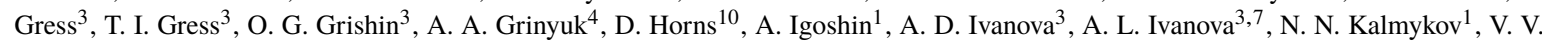
Kindin $^{2}$, S. N. Kiryuhin ${ }^{3}$, R. P. Kokoulin ${ }^{2}$, K. G. Kompaniets ${ }^{2}$, E. E. Korosteleva ${ }^{1}$, V. A. Kozhin ${ }^{1}$, E. A. Kravchenko ${ }^{7}, 8$, A. P. Kryukov ${ }^{1}$, L. A. Kuzmichev ${ }^{1}$, A. A. Lagutin ${ }^{11}$, M. Lavrova ${ }^{4}$, Yu. E. Lemeshev ${ }^{3}$, B. K. Lubsandorzhiev ${ }^{12}$, N. B. Lubsandorzhiev ${ }^{1}$, A. D. Lukanov ${ }^{12}$, D. S. Lukyantsev ${ }^{3}$, S. D. Malakhov ${ }^{3}$, R. R. Mirgazov ${ }^{3}$, R. Mirzoyan ${ }^{13,3}$, R. D. Monkhoev ${ }^{3}$, E. A. Osipova ${ }^{1}$, A. L. Pakhorukov ${ }^{3}$, A. Pan $^{4}$, L. Panasenko $^{7}$, L. V. Pankov ${ }^{3}$, A. D. Panov ${ }^{1}$, A. A. Petrukhin ${ }^{2}$, I. A. Poddubny ${ }^{3}$, D. A. Podgrudkov ${ }^{1}$, V. A. Poleschuk ${ }^{3}$, V. Ponomareva ${ }^{3}$, M. Popesku ${ }^{14}$, E. G. Popova ${ }^{1}$, A. Porelli ${ }^{5}$, E. B. Postnikov ${ }^{1}$, V. V. Prosin ${ }^{1}$, V. S. Ptuskin ${ }^{15}$, A. A. Pushnin ${ }^{3}$, R. I. Raikin ${ }^{11}$, A. Y. Razumov ${ }^{1}$, G. I. Rubtsov ${ }^{12}$, E. V. Ryabov ${ }^{3}$, Y. I. Sagan ${ }^{4,9}$, V. S. Samoliga ${ }^{3}$, A. A. Silaev ${ }^{1}$, A. A. Silaev(junior) ${ }^{1}$, A. Yu. Sidorenkov ${ }^{12}$, A. V. Skurikhin ${ }^{1}$, M. Slunecka ${ }^{4}$, A. V. Sokolov ${ }^{7,8}$, L. G. Sveshnikova ${ }^{1}$, V. A. Tabolenko ${ }^{3}$, A. B. Tanaev ${ }^{3}$, B. A. Tarashansky ${ }^{3}$, M. Yu. Ternovoy ${ }^{3}$, L. G. Tkachev ${ }^{4,9}$, R. Togoo ${ }^{16}$, M. Tluczykont ${ }^{10}$, N. Ushakov ${ }^{12}$, A. Vaidyanathan ${ }^{7}$, P. A. Volchugov ${ }^{1}$, N. V. Volkov ${ }^{11}$, D. Voronin ${ }^{12}$, R. Wischnewski ${ }^{5}$, A. V. Zagorodnikov ${ }^{3}$, D. P. Zhurov ${ }^{3,17}$, I. I. Yashin ${ }^{2}$

${ }^{1}$ Skobeltsyn Institute of Nuclear Physics, Moscow State University, Moscow, 119991 Russia. ${ }^{2}$ National Research Nuclear University MEPhI, Moscow, 115409 Russia. ${ }^{3}$ Research Institute of Applied Physics, Irkutsk State University, Irkutsk, 664003 Russia. ${ }^{4}$ Joint Institute for Nuclear Research, Dubna, Moscow oblast, 141980 Russia. ${ }^{5}$ Deutsches Elektronen-Synchrotron DESY, Zeuthen, 15738 Germany. ${ }^{6}$ Physics Department of the University of Torino and Istituto Nazionale di Fisica Nucleare, Torino, 10125 Italy. ${ }^{7}$ Novosibirsk State University, Novosibirsk, 630090 Russia. ${ }^{8}$ Budker Institute of Nuclear Physics, Siberian Branch, Russian Academy of Sciences, Novosibirsk, 630090 Russia. ${ }^{9}$ Dubna University, Dubna, Moscow oblast, 141980 Russia. ${ }^{10}$ Institut für Experimentalphysik, Universität Hamburg, Hamburg, D-22 761 Germany. ${ }^{11}$ Altai State University, Barnaul, Altai krai, 656049 Russia. ${ }^{12}$ Institute for Nuclear Research, Russian Academy of Sciences, Moscow, 117312 Russia. ${ }^{13}$ Max Planck Institute for Physics, Munich, 80805 Germany. ${ }^{14}$ Space Science Institute, Magurele, 077125 Romania. ${ }^{15}$ Pushkov Institute of Terrestrial Magnetism, Ionosphere and Radio Wave Propagation, Russian Academy of Sciences, Troitsk, Moscow, 142190 Russia. ${ }^{16}$ Institute of Physics and Technology Mongolian Academy of Sciences, Ulaanbaatar, Mongolia. ${ }^{17}$ Irkutsk National Research Technical University, Irkutsk, Russia. 